\title{
MIRADAS for the Gran Telescopio Canarias: System Overview
}

S.S. Eikenberry, J.G. Bennett, B. Chinn, H.V. Donoso, S.A. Eikenberry, E. Ettedgui, A. Fletcher, R. Frommeyer, A. Garner, M. Herlevich, N. Lasso, P. Miller, S. Mullin, C. Murphey, S.N. Raines, C. Packham, S. Schofield, R.D. Stelter, F. Varosi, C. Vega, C. Warner

Department of Astronomy, University of Florida, Gainesville, FL 32611

F. Garzón , J. Rosich

Instituto Astrofisica de Canarias, La Laguna, Tenerife, Spain

J.M. Gomez, J. Sabater, C. Vilar, J. Torra

Universidad de Barcelona, Barcelona, Spain

J. Gallego, N. Cardiel. C. Eliche, S. Pascual

Universidad Complutense de Madrid, Madrid, Spain

O. Ballester, J.M. Illa, J. Jimenez, L. Cardiel-Sas

Institut de Física d'Altes Energies, Barcelona, Spain

J. Galipienzo, M.A. Carrera

Added Value Solutions, Eibar, Spain

P. Hammersley

European Southern Observatory, Garching, Germany

Salvador Cuevas

Instituto de Astronomia, Universidad Nacional Autonoma de Mexico, Mexico D.F.

\begin{abstract}
The Mid-resolution InfRAreD Astronomical Spectrograph (MIRADAS, a near-infrared multi-object echelle spectrograph operating at spectral resolution $\mathrm{R}=20,000$ over the 1-2.5 $\mu \mathrm{m}$ bandpass) was selected in 2010 by the Gran Telescopio Canarias (GTC) partnership as the next-generation near-infrared spectrograph for the world's largest optical/infrared telescope, and is being developed by an international consortium. The MIRADAS consortium includes the University of Florida, Universidad de Barcelona, Universidad Complutense de Madrid, Instituto de Astrofísica de Canarias, Institut de Física d'Altes Energies, Institut d'Estudis Espacials de Catalunya and Universidad Nacional Autonoma de Mexico, as well as probe arm industrial partner A-V-S (Spain). In this paper, we review the overall system design for MIRADAS, as it nears Preliminary Design Review in the autumn of 2012.
\end{abstract}

\section{INTRODUCTION}

The Mid-resolution InfRAreD Astronomical Spectrograph (MIRADAS) for GTC is the most powerful astronomical instrument of its kind ever developed. The combination of the collecting area of GTC and the multi-object midresolution near-infrared spectra provided by MIRADAS make its capabilities unparalleled for addressing some of the leading scientific challenges of the coming decades. The MIRADAS Science Working Group has identified 4 key science cases for MIRADAS. These 4 key cases form the "Design Reference Cases" for MIRADAS - the scientific performance capabilities which provide the fundamental requirements drivers for the instrument. In this paper, we

Ground-based and Airborne Instrumentation for Astronomy IV, edited by lan S. McLean, Suzanne K. Ramsay, Hideki Takami, Proc. of SPIE Vol. 8446, 844657 - ( ) 2012 SPIE · CCC code: 0277-786X/12/\$18 - doi: 10.1117/12.925686 
present the basic instrument overview, which covers the basic concept of the instrument intended to meet the requirements of the Design Reference Cases.

The basic MIRADAS concept is a near-infrared multi-object echelle spectrograph operating at spectral resolution $\mathrm{R}=20,000$ over the 1-2.5 $\mu \mathrm{m}$ bandpass. MIRADAS selects targets using 20 deployable probe arms with pickoff mirror optics, each feeding a 3.7x1.2-arcsec field of view to the spectrograph. The spectrograph input optics also include a "slit slicer" which reformats each probe field into 3 end-to-end slices of a fixed 3.7x0.4-arcsec format - combining the advantages of minimal slit losses in any seeing conditions better than 1.2-arcsec, while at the same time providing some (limited) two-dimensional spatial resolution. The spectrograph optics then provide a range of configurations providing the observer with the ability to choose between maximal multiplex advantage and maximal wavelength coverage, with several intermediate options, depending upon the needs of the science program. Its basic parameters are as follows:

Table 1 - General Parameters

\begin{tabular}{|l|c|l|}
\hline Parameter & Value & Comment \\
\hline Target field of regard & 5-arcminute diameter & $\begin{array}{l}\text { Each probe arm patrols a "slice of } \\
\text { pie" wedge of this circular field }\end{array}$ \\
\hline Individual target field of view & $3.7 \times 1.2$-arcsec & \\
\hline Slit slicer geometry & 3 slices of 3.7x0.4-arcsec ea. & \\
\hline Detector focal plane & $4096 \times 2048$ pixels & Mosaic of 2Kx2K HAWAll-2RG \\
\hline Spectro-polarimetry & Linear, circular & $\begin{array}{l}\text { Available for single-object cross- } \\
\text { dispersed mode }\end{array}$ \\
\hline Continuum sensitivity & $\begin{array}{l}\mathrm{J}=18.0 \mathrm{mag} \\
\mathrm{H}=17.4 \mathrm{mag} \\
\mathrm{K}=16.7 \mathrm{mag}\end{array}$ & $\begin{array}{l}\mathrm{S} / \mathrm{N}=10 \text { for 1-hour on-source } \\
\text { exposure }\end{array}$ \\
\hline Emission line sensitivity & $5 \times 10^{-18} \mathrm{ergs} / \mathrm{cm}^{2} / \mathrm{s}$ (point) & $\begin{array}{l}\mathrm{S} / \mathrm{N}=10 \text { for 1-hour on-source } \\
\text { exposure; resolved source } \\
\text { assumes 1 square arcsecond } \\
\text { detect cell }\end{array}$ \\
\hline
\end{tabular}

Table 2 - Multiplex Configurations

\begin{tabular}{|l|c|l|}
\hline \multicolumn{1}{|c|}{ Configuration } & Number of MXS Targets & \multicolumn{1}{c|}{ Instantaneous Bandpass } \\
\hline SO-mode short & 1 & $0.98-1.80 \mu \mathrm{m}$ \\
\hline SO-mode long & 1 & $1.287-2.50 \mu \mathrm{m}$ \\
\hline Medium MXS J & 5 & $1.14-1.32 \mu \mathrm{m}$ \\
\hline Medium MXS H & 5 & $1.50-1.82 \mu \mathrm{m}$ \\
\hline Medium MXS K & 5 & $1.92-2.49 \mu \mathrm{m}$ \\
\hline Maximum MXS & 20 & Any SINGLE order from Table 3 \\
\hline
\end{tabular}




\section{Table 3 - MIRADAS Echelle Orders}

\begin{tabular}{|c|c|c|c|c|c|c|}
\hline Order & $\begin{array}{c}\text { Wavelength } \\
(\boldsymbol{\mu \mathbf { m } )}\end{array}$ & Band & & Order & $\begin{array}{c}\text { Wavelength } \\
(\boldsymbol{\mu \mathbf { m }})\end{array}$ & Band \\
\hline 14 & $2.390-2.500$ & $\mathrm{~K}$ & & 24 & $1.394-1.479$ & - \\
\hline 15 & $2.231-2.366$ & $\mathrm{~K}$ & & 25 & $1.339-1.420$ & $\mathrm{~J}$ \\
\hline 16 & $2.092-2.218$ & $\mathrm{~K}$ & & 26 & $1.287-1.365$ & $\mathrm{~J}$ \\
\hline 17 & $1.968-2.087$ & $\mathrm{~K}$ & & 27 & $1.239-1.314$ & $\mathrm{~J}$ \\
\hline 18 & $1.859-1.971$ & - & & 28 & $1.195-1.267$ & $\mathrm{~J}$ \\
\hline 19 & $1.761-1.868$ & $\mathrm{H}$ & & 29 & $1.154-1.224$ & $\mathrm{~J}$ \\
\hline 20 & $1.673-1.774$ & $\mathrm{H}$ & & 30 & $1.115-1.183$ & $\mathrm{~J}$ \\
\hline 21 & $1.593-1.690$ & $\mathrm{H}$ & & 31 & $1.079-1.448$ & $\mathrm{~J}-\mathrm{lo}$ \\
\hline 22 & $1.521-1.613$ & $\mathrm{H}$ & & 32 & $1.046-1.109$ & $\mathrm{~J}-\mathrm{lo}$ \\
\hline 23 & $1.455-1.543$ & - & & 33 & $1.014-1.075$ & $\mathrm{~J}-\mathrm{lo}$ \\
\hline & & & & 34 & $0.984-1.044$ & $\mathrm{~J}-\mathrm{lo}$ \\
\hline
\end{tabular}

We present a conceptual layout (“cartoon" sketch) for MIRADAS in Figure 1, and the resulting scientific grasp comparison for the instrument in Figure 2. In Figure 3, we show the evolution of the MIRADAS focal planes. 


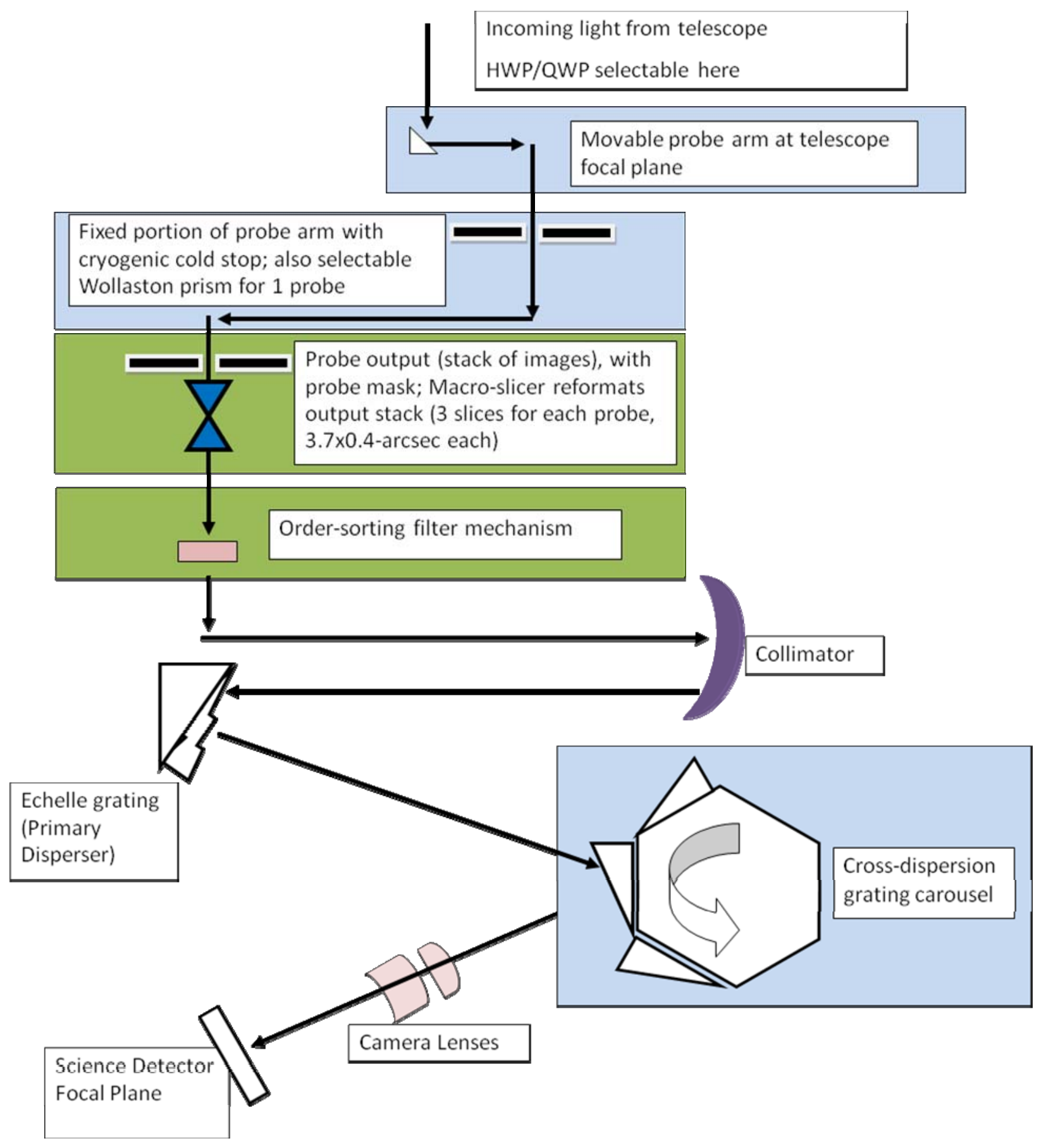

Figure 1 - MIRADAS Conceptual Layout Schematic 


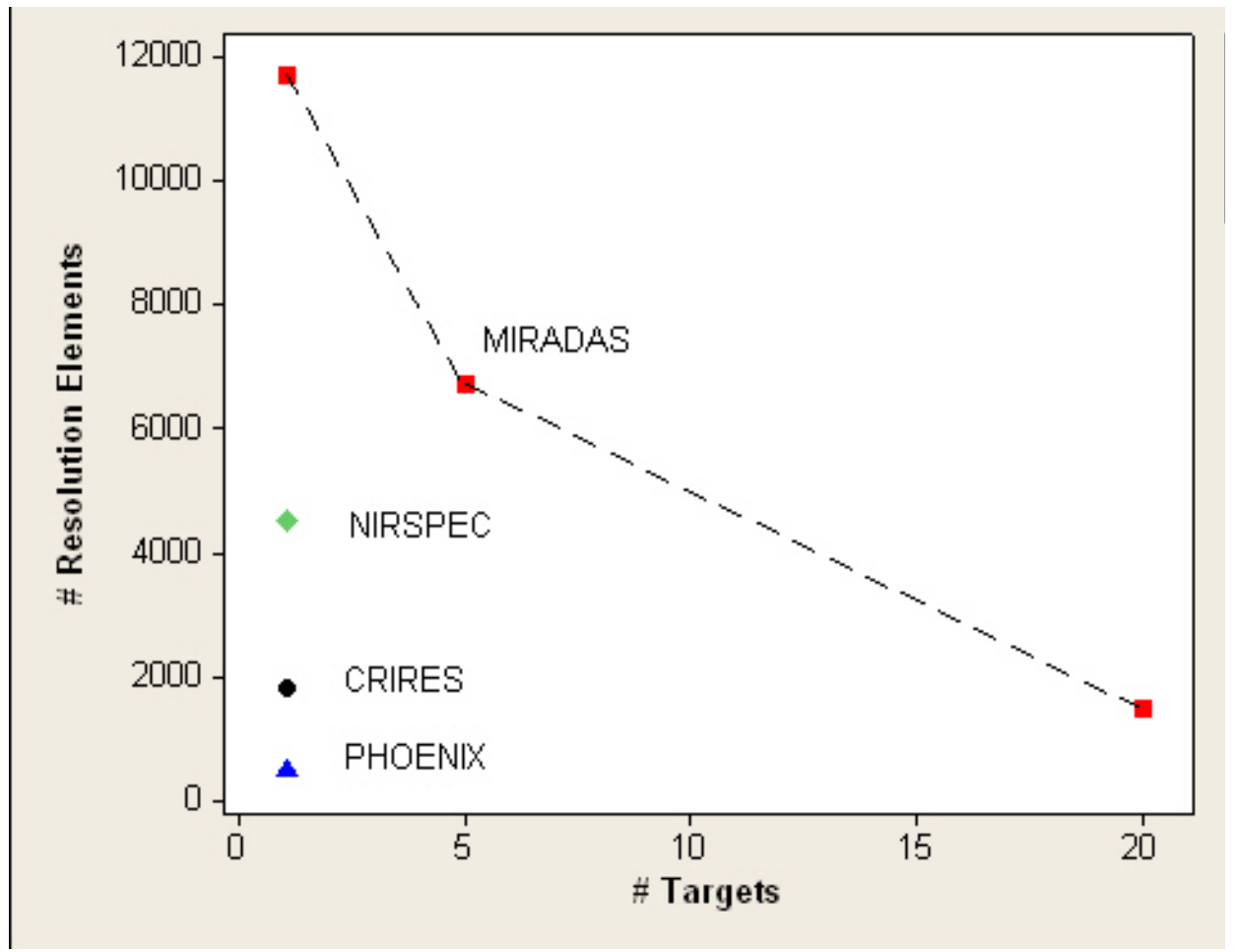

Figure 2: MIRADAS scientific grasp (instantaneous number of targets and spectral resolution elements per target) compared to other 8-m-class spectrographs with $R=20,000$ or higher. Note that MIRADAS is the only multi-object spectrograph in this class, and it provides more spectral information for each of its $N=5$ targets than others do for $N=1$ target. 


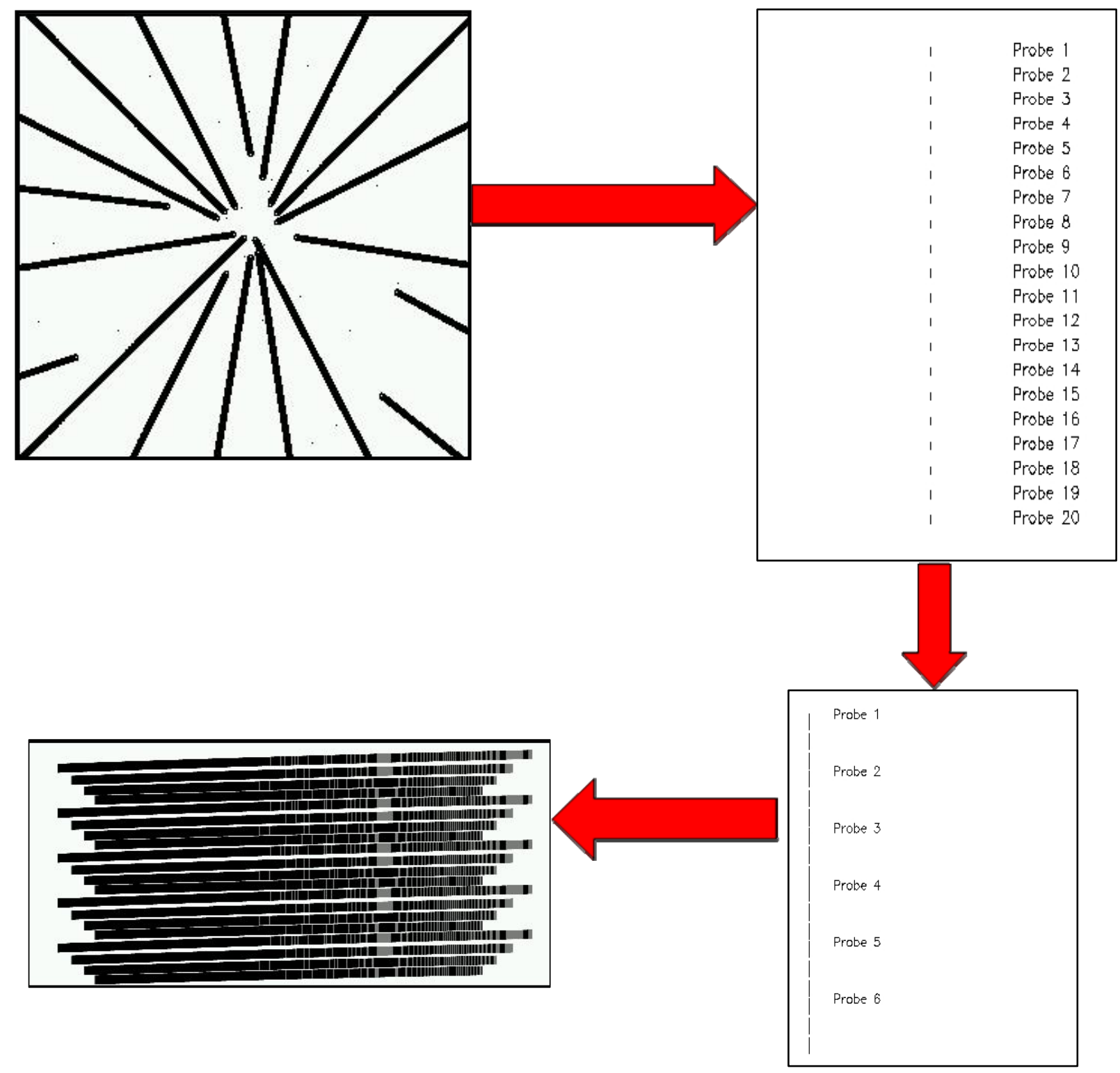

Figure 3: MIRADAS focal plane evolution. (Top left) Schematic view of MXS probe arms selecting targets within the 5-arcmin field of regard at the GTC focal plane; (Top-right) Schematic view of the 20 probe outputs at the second focal plane (input to the macroslicer); (Bottom-right) Schematic view of a section of the "sliced" probe outputs forming the pseudo-longslit at the third focal plane (spectrograph input slit); (Bottom-left) Cross-dispersed 5-target K-band spectra at the final focal plane (science detectors).

\section{KEY TECHNOLOGIES: PROBE ARMS}

MIRADAS includes a multiplexing system (MXS) employing 20 deployable probe arms with pickoff mirrors. MIRADAS MXS probe arms, based on previous conceptual designs for HRNIRS (Muller et al., 2006; Eikenberry et al., 2006a; Hinkle et al., 2006; Liang et al., 2006) and IRMOS (Eikenberry et al., 2006b; Andersen at al., 2006) for the Gemini Observatories.

The MXS patrol field for MIRADAS is 5-arcminutes in diameter, or about 250-mm. The primary function of the MXS probe mechanism is to select target fields-of-view and relay light from them to the rest of the spectrograph. This is accomplished using 20 independently-controlled/actuated MXS pick-off probe arms. Each arm patrols a sector ("slice of pie") of the MIRADAS field, just above the input telescope focal plane. Optics in each arm relay light from a 3.7x1.2- 
arcsec field. Figure 4 shows a view of the MXS probe mechanism. The 20 probe arms each patrol an 18-degree "slice of pie" region just above this focal plane, relaying light to an output focal plane in a pseudo-longslit. A pickoff mirror located near the telescope focal plane relays light down the probe arm, where it encounters a collimating doublet lens. The lens feeds light through a series of folds in the probe mechanism, which maintain a fixed optical path length while the probe arm is moved to variable target locations in the field of regard. The $4^{\text {th }}$ fold mirror is under the MXS optical bench, and is fixed in its location and orientation. Shortly after the fixed fold comes the cold pupil stop, located at the pupil image created by the collimator doublet. Finally, a re-imaging doublet brings the beam to a focus.
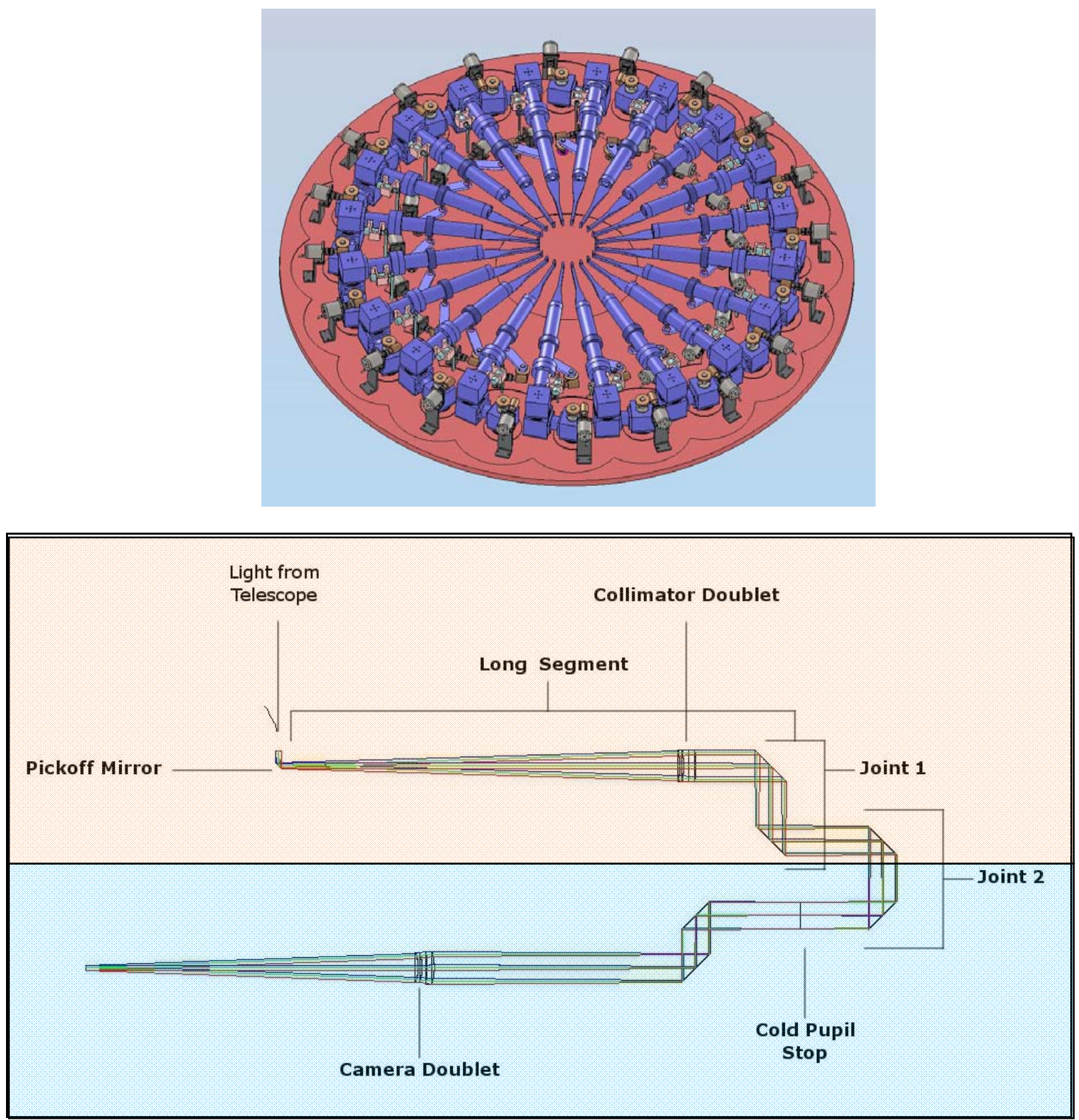

Figure 4-MIRADAS MXS probe optical layout. The top (pink) shaded portion includes the moving parts of the probe arm, while the bottom (blue) shaded portion includes the fixed parts of the probe arm optical train. 
At the output focal plane from the MXS system, the individual probes produce fields of 3.7.0x1.2-arcsec (2.7x0.8-mm), each of which is separated from the next probe by a 10.3-mm center-to-center distance, creating a sparsely-filled pseudolongslit at the macro-slicer input.

In Figure 5, we present a more detailed view of a single probe arm mechanical model, as well as the first prototype probe arm (P1A) constructed at UF in 2012. An identical prototype (P1B) was constructed at AVS in Spain and is undergoing testing at the University of Barcelona. The P1A unit has been extensively tested under warm conditions at UF, showing that it meets all basic requirements and has excellent RMS repeatability (Figure 6). A cryogenic prototype (P2) is currently under development as well.
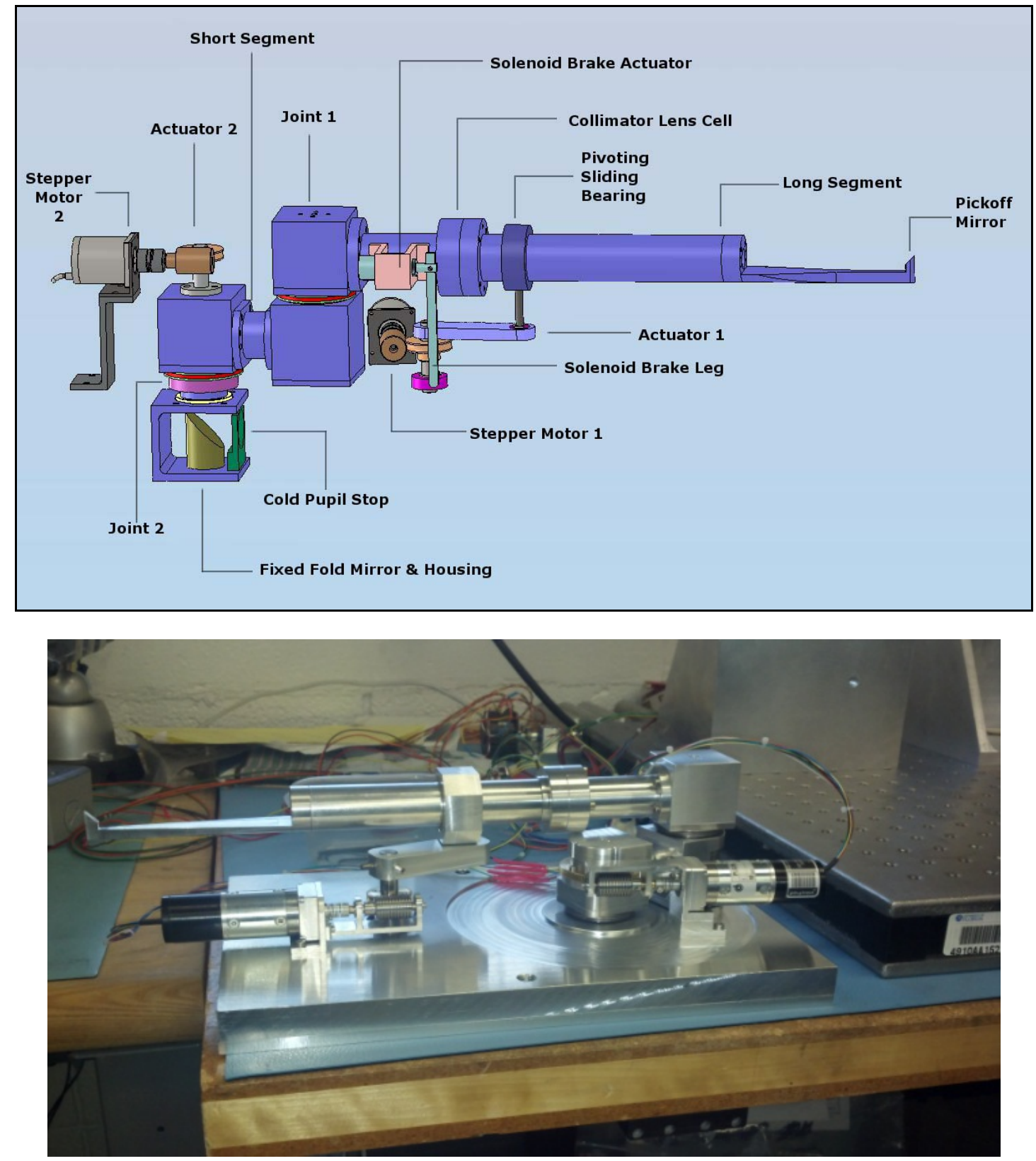

Figure 5 - (Top) Detailed side view of a single MIRADAS probe arm. (Bottom) Photo of Prototype 1A at the University of Florida. 

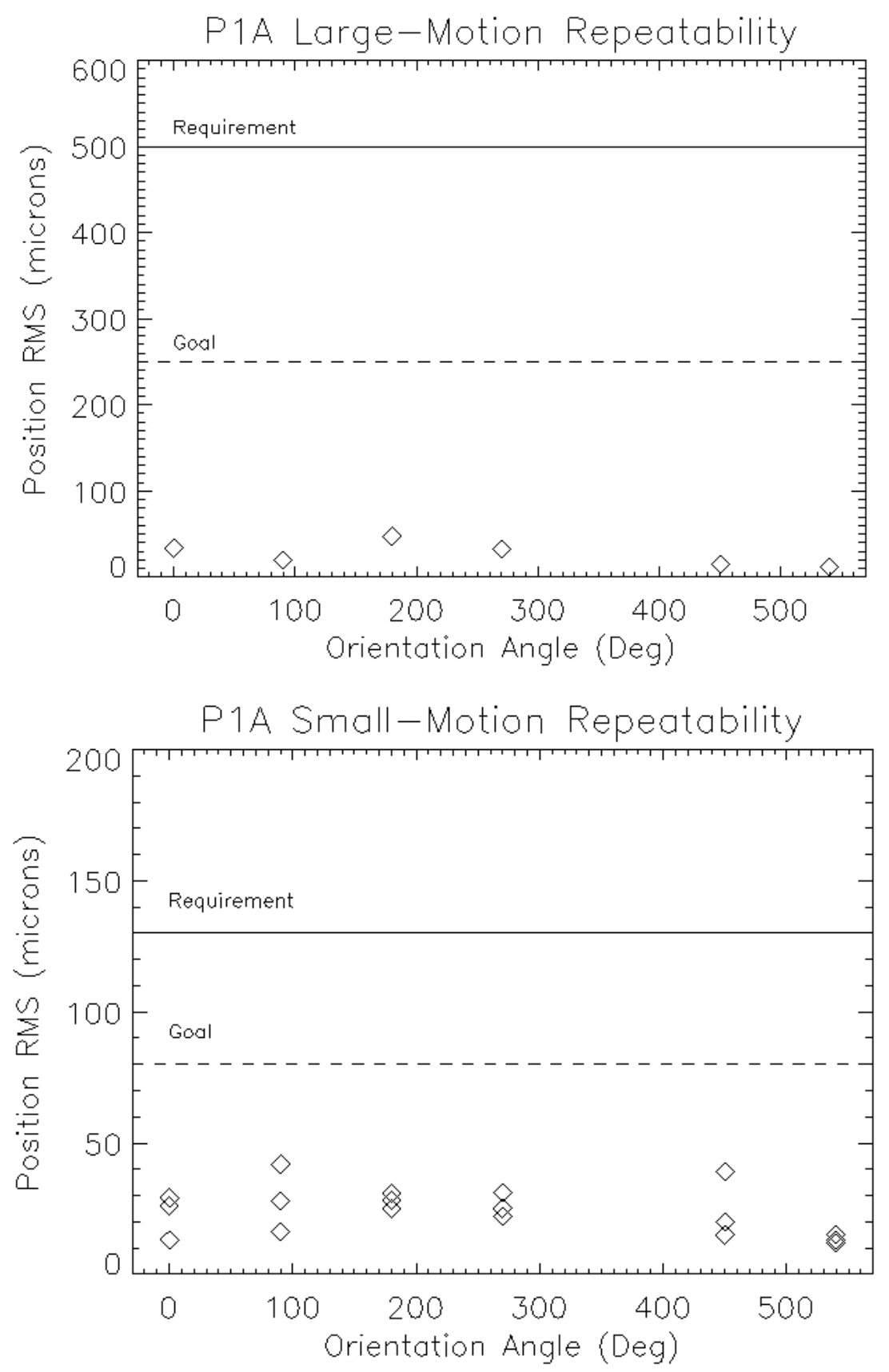

Figure 6 - Measured repeatability performance of probe arm motions for the Prototype $1 A$ probe arm. Note that the arm RMS is MUCH better than the requirement and goal performance established for both large and small motions.

\section{KEY TECHNOLOGIES: MACRO-SLICER}

The next key component for MIRADAS is the "macro-slicer". This is an image slicing mini-IFU which takes the input focal plane (a 200x0.8-mm sparsely-filled pseudo-longslit) and slices it into a different, nearly-filled, pseudo-longslit of length $\sim 80.5 \times 0.135-\mathrm{mm}$ (i.e. dividing each slitlet into 3 narrower slitlets oriented lengthwise). This is the same approached used for the FISICA and FRIDA image slicers. 
The input focal plane of the MIRADAS macro-slicer differs significantly from that of the previous slicer IFUs such as FISICA and FRIDA - not only in it narrow aspect ratio, but also in the length of the field ( $\sim 200$-mm versus $\sim 20-30$-mm for FISICA/FRIDA). For typical previous slicers, the curvature of the slicer mirror itself would introduce significant defocus issues over such a long distance. However, the MIRADAS macro-slicer input also differs from the previous IFUs in that its input field is NOT a continuous telescopic focal image, but is in fact a sparsely-filled field composed of disjoint relay inputs. As such, we can freely "segment" the slicer array into sub-slicers - each with the required width, but much shorter length (thus reducing the mirror curvature defocus issue) and with the between-segment gaps chosen to lie between the disjoint probe fields. We thus separate the slicer mirror into 5 slicer segments, each of which covers 4 MXS probe inputs. The slicer length is thus reduced to 35-mm - very similar to the values used for FRIDA and FISICA, and thus reducing the curvature problem to being nearly negligible.

From that point on, the macro-slicer more closely resembles the previous slicer IFUs built at the University of Florida, with each slice being mapped to a pupil mirror in an array, and from there focused onto an output focal plane in the form of a nearly-filled pseudo-longslit.

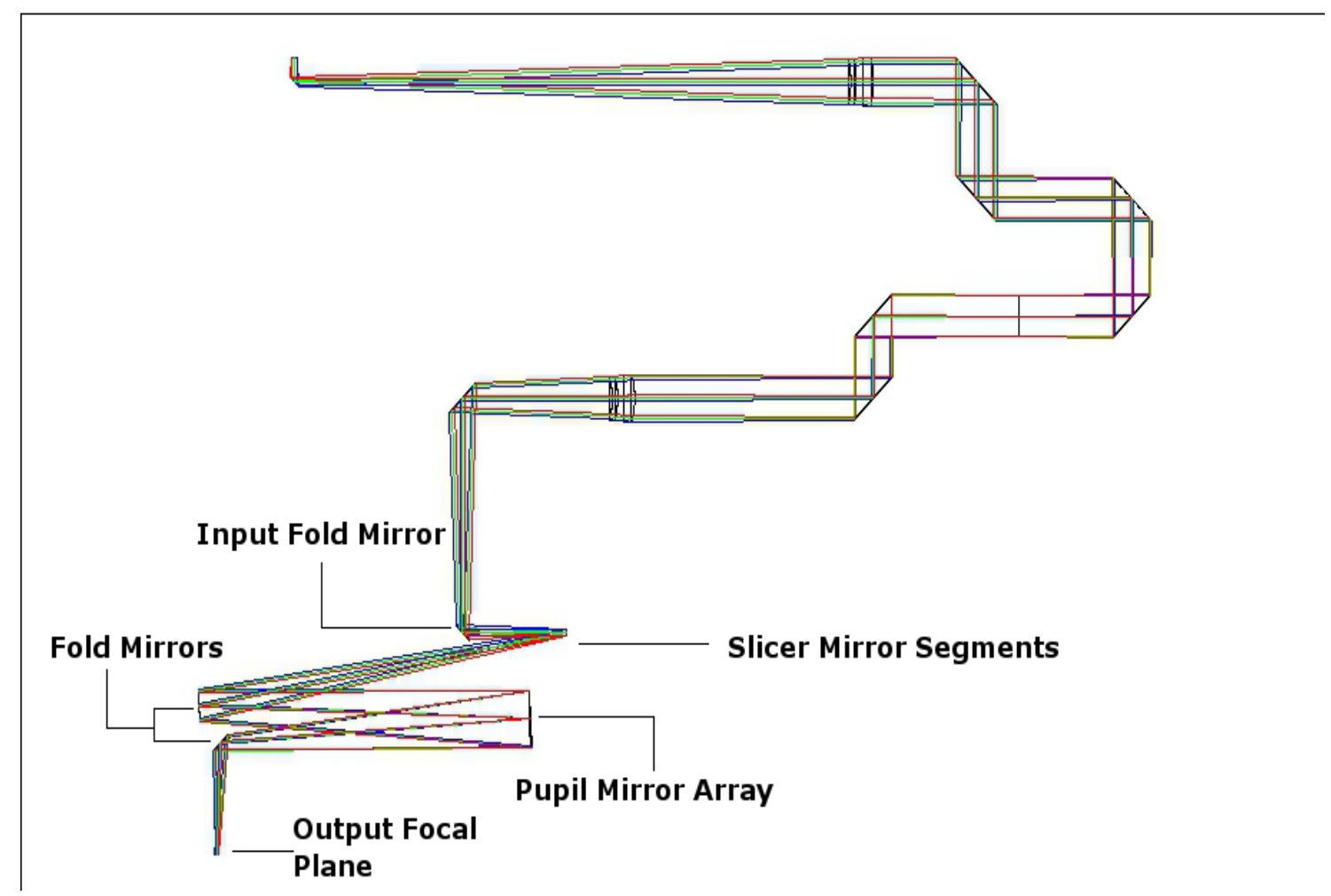

Figure 7 - Probe arm and macro-slicer ZEMAX layout

\section{SPECTRAL MODES AND FORMATS}

For the various modes described above, MIRADAS provides different spectral layouts and formats on the 4096x2048pixel detector mosaic. We present examples of those modes $(\mathrm{N}=1, \mathrm{~N}=5, \mathrm{~N}=20)$ those graphically here. 


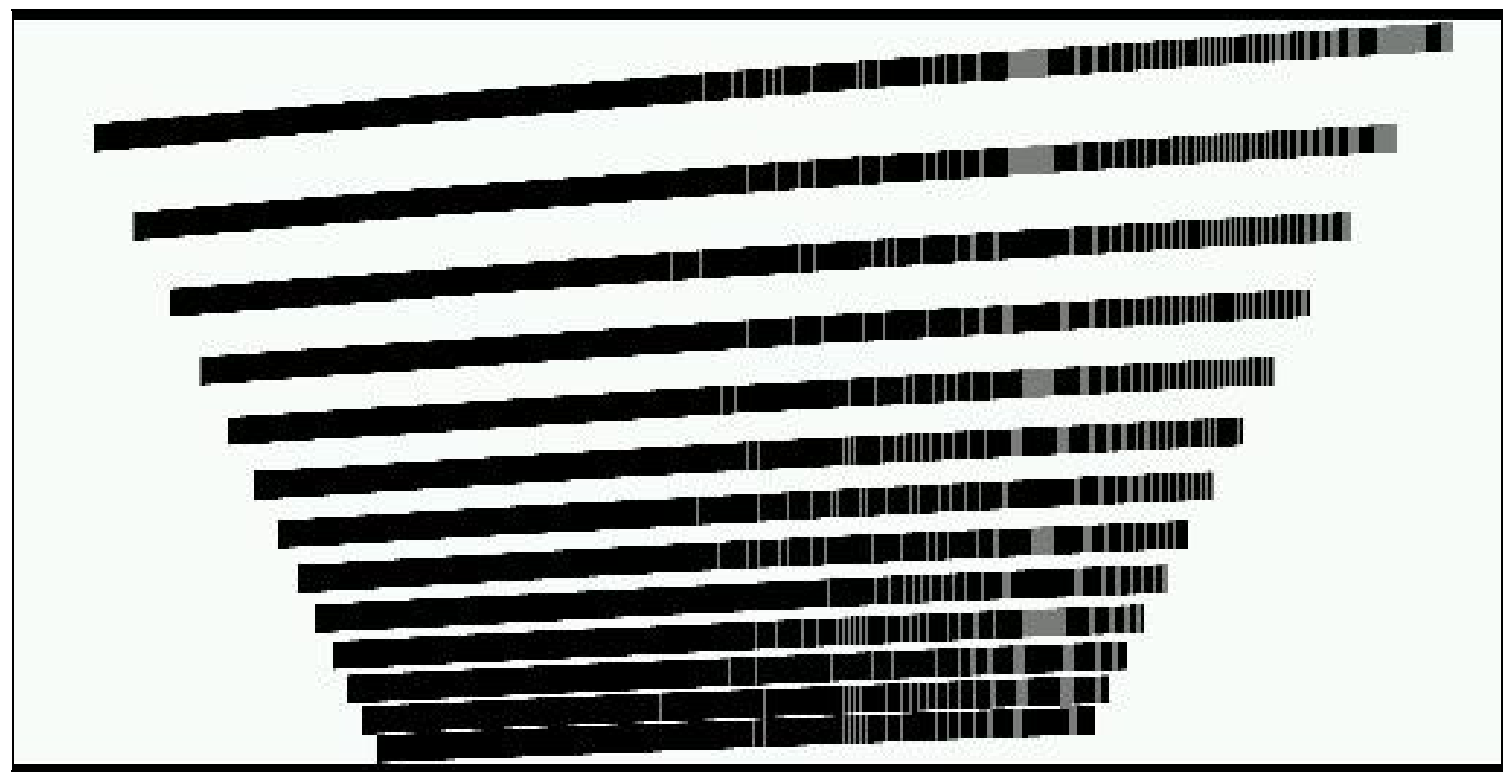

Figure 8 - MIRADAS single-object long-wavelength mode cross-dispersed spectrum projected on the 4096x2048-pixel detector mosaic. The spectrum covers the wavelength range from 1.28-2.50 $\mu \mathrm{m}$ (orders 14-26 from top to bottom).

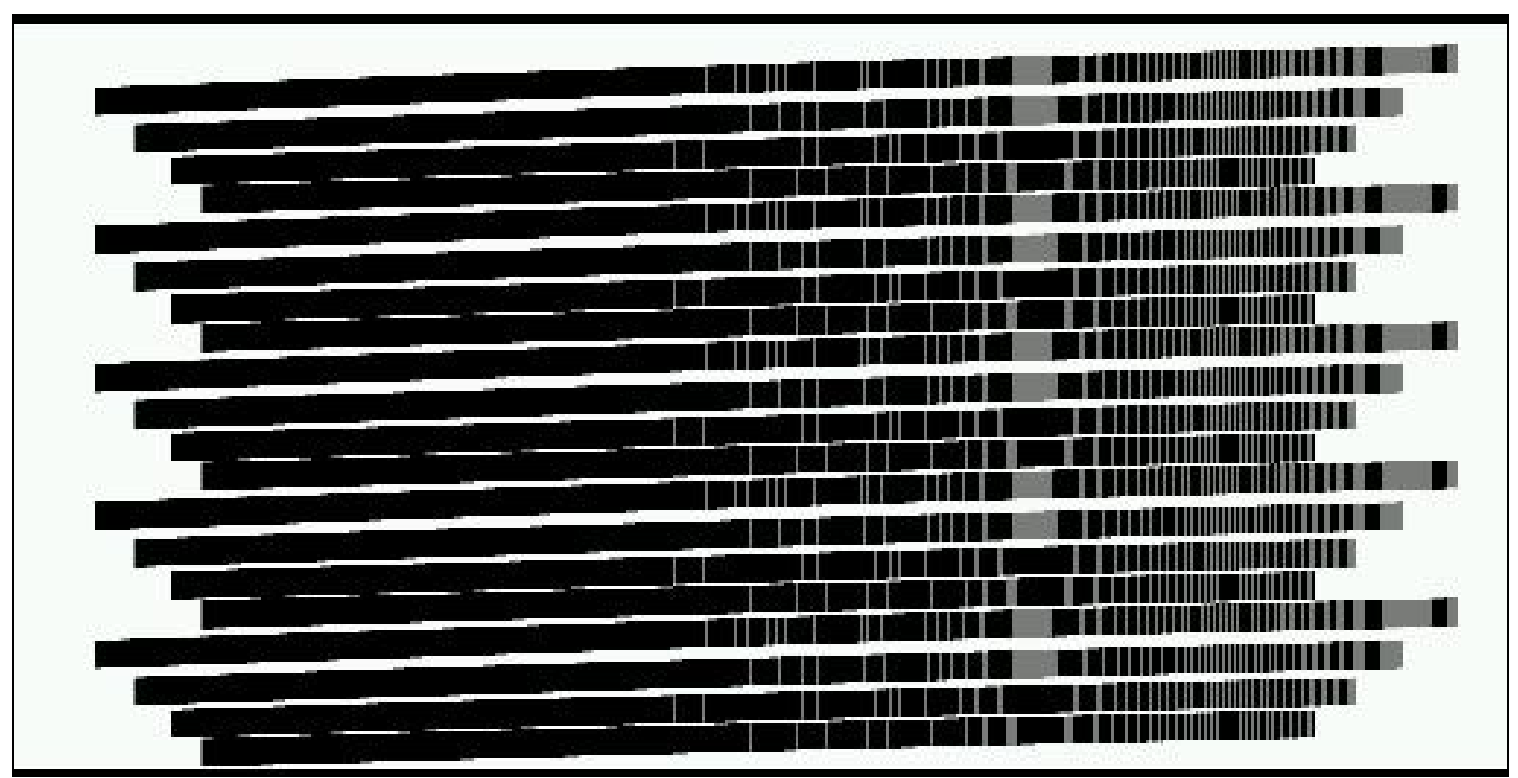

Figure 9 - MIRADAS medium-multiplex K-band mode cross-dispersed spectra projected on the 4096x2048-pixel detector mosaic. The spectrum covers the wavelength range from 1.92-2.50 $\mu \mathrm{m}$ (orders 14-17) for 5 separate science targets simultaneously. 


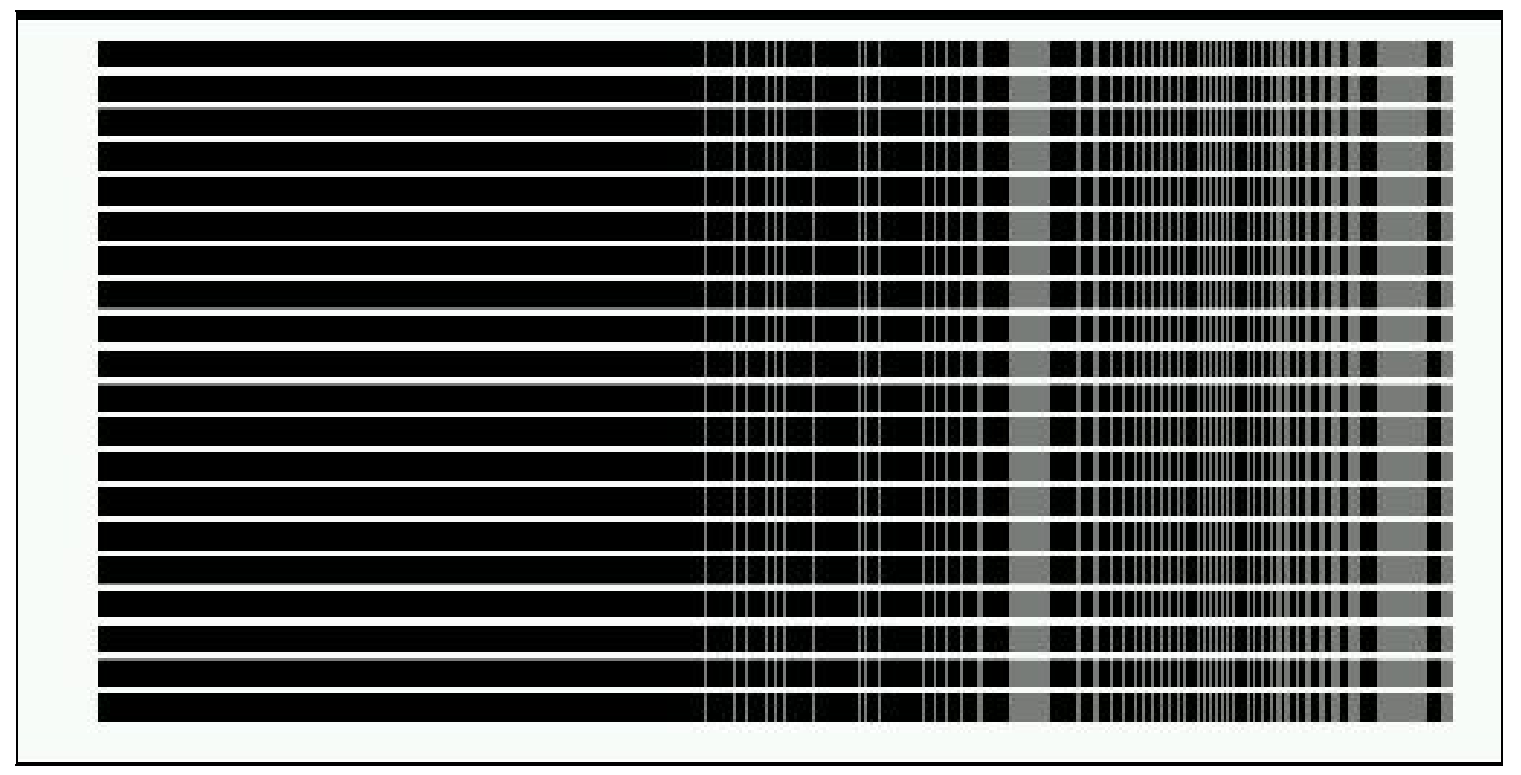

Figure 10 - MIRADAS maximum-multiplex mode spectra projected on the 4096x2048-pixel detector mosaic. The spectrum covers the wavelength range of any single order (order shown is 14) for 20 separate science targets simultaneously.

\section{DETECTOR ELECTRONICS AND DATA PIPELINE}

MIRADAS will use two Teledyne HAWAII-2RG arrays in a 4096x2048-pixel mosaic. Two array control options are under study and development. The first, being led by IFAE with support at UF, is a modified MONSOON controller. The second, being led by UF with support at IFAE, is a SIDECAR interface (ISDEC) developed at IUCAA (India) (see Ramaprakash, 2010 for details) and used for the CIRCE instrument (S. Eikenberry - PI). We show early test results from the ISDEC controller with a H2RG multiplexer in Figure 11.
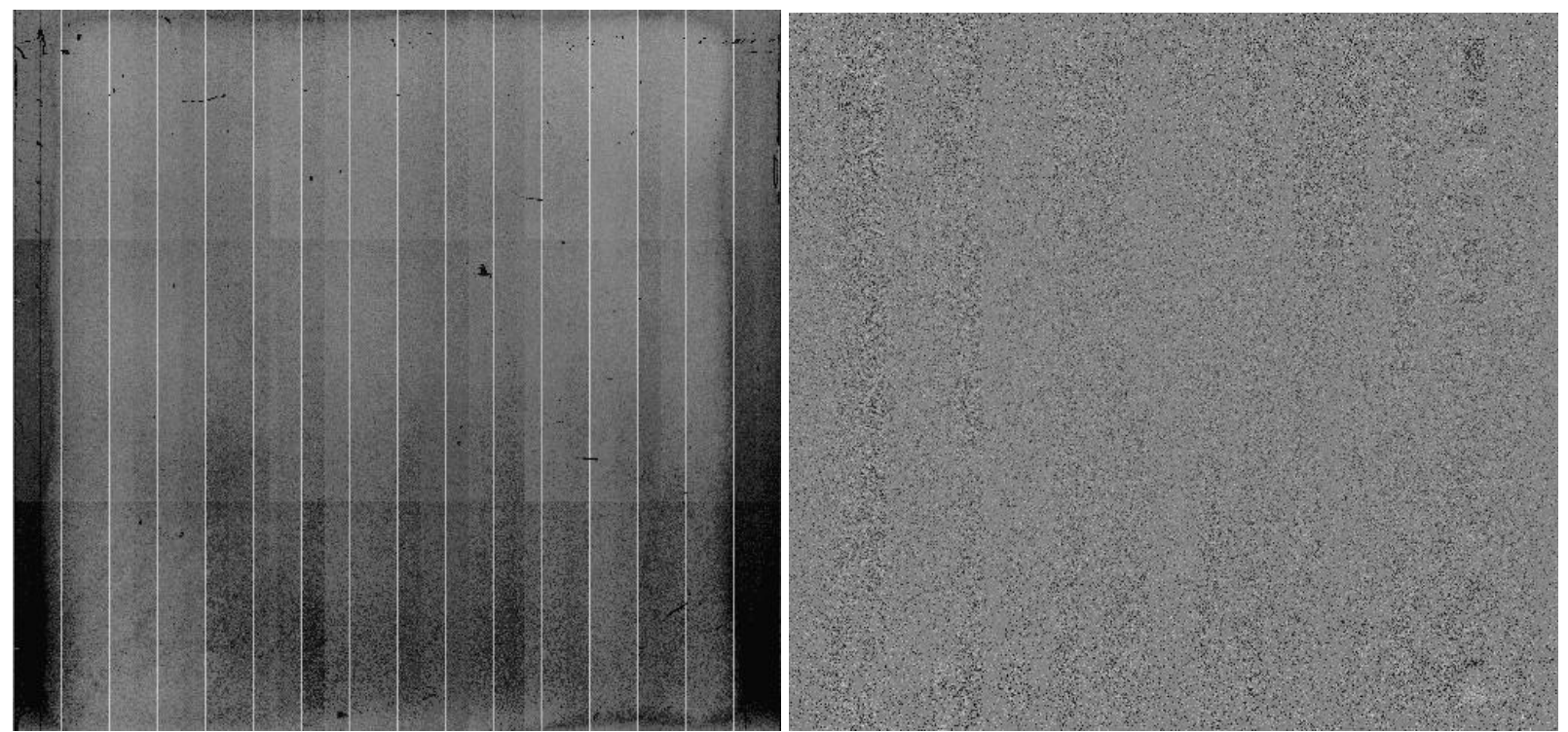

Figure 11 - (Left) Raw single read of a HAWAII-2RG warm multiplexer with ISDEC, taken in the lab at UF. (Right) CDS image of the same. 
The MIRADAS data reduction pipeline is being developed at UCM, and is based on the Python pipeline experience there for previous GTC infrared instruments. The MIRADAS data pipeline will provide fully reduced and calibrated data for all modes of the instrument, including spectro-polarimetry.

\section{INSTRUMENT CRYOSTAT AND ENVELOPE}

The MIRADAS instrument conforms to the GTC envelope of mass/volume for the Folded Cassegrain focus. The protruding coldheads (Figure 12) require a variance, but do not interfere with other components/instruments on the telescope.
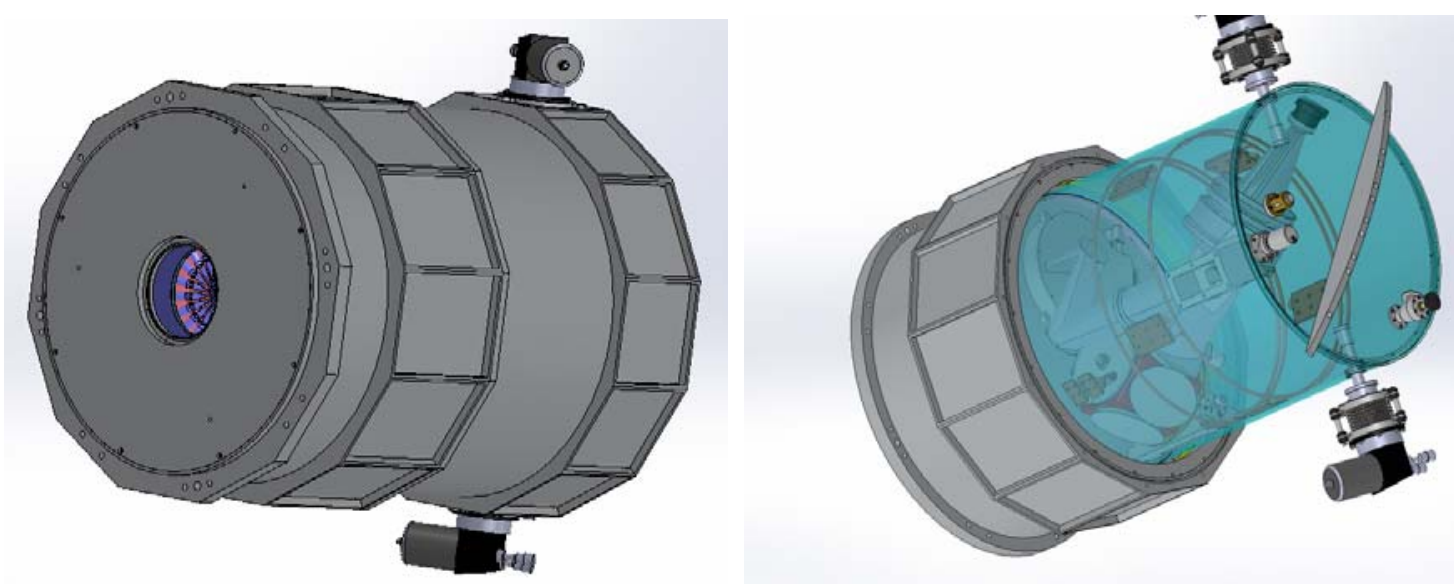

Figure 12 - MIRADAS instrument views. (Left) MIRADAS full instrument view. (Right) Same, but with rear vacuum jacket removed.

\section{REFERENCES}

Andersen, David R.; Eikenberry, Stephen S.; Fletcher, Murray; Gardhouse, William; Leckie, Brian; Véran, Jean-Pierre; Gavel, Don; Clare, Richard; Guzman, Rafael; Jolissaint, Laurent; Julian, Roger; Rambold, William, 2006, Proc. Of SPIE, Vol. 6269, 145

Eikenberry, Stephen; Hinkle, Kenneth; Joyce, Dick; Liang, Ming; Muller, Gary; Heileman, Ed; French, Jim; Ge, Jian; Packham, Chris; Julian, Roger; Gaughan, Neil; Sprayberry, David, 2006a, Proc. Of SPIE, Vol. 6271, 31E

Eikenberry, Stephen; Andersen, David; Guzman, Rafael; Bally, John; Cuevas, Salvador; Fletcher, Murray; Gardhouse, Rusty; Gavel, Don; Gonzalez, Anthony; Gruel, Nicolas; Hamann, Fred; Hamner, Sam; Julian, Roger; Julian, Jeff; Koo, David; Lada, Elizabeth; Leckie, Brian; Lopez, J. Alberto; Pello, Roser; Perez, Jorge; Rambold, William; Roman, Carlos; Sarajedini, Ata; Tan, Jonathan; Venn, Kim; Veran, Jean-Pierre; Ziegert, John, 2006b, Proc. Of SPIE, Vol. 6269, 188

Hinkle, Kenneth H.; Eikenberry, Stephen; Joyce, Richard; Liang, Ming; Muller, Gary; Hileman, Ed; French, Jim; Ge, Jian; Packham, Chris; Julian, Roger; Armandroff, Taft; Gaughan, Neil; Sprayberry, David, 2006, Proc. Of SPIE, Vol. 6269, 117

Liang, Ming; Joyce, R. R.; Eikenberry, S. S.; Hinkle, K. H.; Muller, G. P.; Ge, Jian; Sprayberry, David, 2006, Proc. Of SPIE, Vol. 6269, 139

Muller, G. P.; Hileman, E. A.; Hinkle, K. H.; Joyce, R. R.; Liang, M.; Sprayberry, D.; Eikenberry, S. S., 2006, Proc. Of SPIE, Vol. 6269, 142

Ramaprakash, Anamparambu; Burse, Mahesh; Chordia, Pravin; Chillal, Kalpesh; Kohok, Abhay; Mestry, Vilas; Punnadi, Sujit; Sinha, Sakya, 2010, Proc. of SPIE, Vol. 7742, 68 